\title{
ERGONOMIA E AS ATIVIDADES OCUPACIONAIS DA EQUIPE DE ENFERMAGEM
}

\section{ERGONOMICS AND THE OCCUPATIONAL ACTIVITIES OF THE NURSING STAFF}

Neusa Maria Costa Alexandre*

ALEXANDRE, N.M.C. Ergonomia e as ativividades ocupacionais da equipe de enfermagem. Rev.Esc.Enf.USP, v.32, n.1, p.84-90, abr. 1998.

\section{RESUMO:}

Esse trabalho discute determinadas condições ergonômicas do trabalho que causam lesões no sistema músculoesquelético da coluna vertebral, relacionando-as com as atividades ocupacionais da equipe de enfermagem.

UNITERMOS: Ergonomia. Atividades ocupacionais. Coluna vertebral.

\section{ABSTRACT:}

This paper discusses some of the ergonomics conditions that contribute to the development of musculoskeletal disorders of the vertebral column and relates these conditions: to the occupational activities of the nursing staff.

UNITERMS: Ergonomics. Occupational activity. Vertebral column.

\section{1 - INTRODUÇÃO}

A questão dos efeitos da ocupação sobre a saúde de trabalhadores tem mobilizado pesquisadores e organizações preocupados com questões relativas à saúde e trabalho ${ }^{38,50,51,54,64}$.

Cada vez mais procura-se estudar como a ocupação pode comportar-se como importante determinante na produção de certas doenças. Nestas circunstâncias, as lesões do sistema músculoesquelético têm despertado a atenção de pesquisadores do mundo inteiro, visto que são uma das mais importantes causas de morbidade e de incapacidade de adultos, e também pelos custos econômicos que acarretam ${ }^{4,46}$.

A elucidação de fatores que causam lesões no sistema osteomuscular tem sido objeto de numerosos trabalhos que atualmente se voltam para aspectos amplos como fatores psicologicos, condições socioeconômicas, defeitos posturais e estilo de vida. Especificamente em relação às atividades profissionais, sabe-se que esse sistema pode ser agredido por fatores relacionados ao levantamento e transporte de cargas, às condições ambientais do posto de trabalho e à própria organização do trabalho, entre outros ${ }^{5,46,48,64}$.

Determinadas posturas e movimentações adotadas por um trabalhador repetidamente, durante anos, pode afetar a sua musculatura e a sua constituição ósseo-articular, principalmente a da coluna e dos membros, resultando, em curto prazo, em dores que se prolongam além do horário de trabalho. Em longo prazo podem resultar em lesões permanentes e deformidades ${ }^{527,29,33}$.

Entre essas afecções estão incluídas as enfermidades da coluna vertebral sendo que as dores e queixas crônicas relacionadas com a coluna vertebral constituem um complexo desafio para a Saúde Ocupacional.

É importante ressaltar que o desenvolvimento de problemas na coluna vertebral é mais comum em determinadas ocupações, estando associado especialmente a fatores ergonômicos e traumáticos ${ }^{64}$

\footnotetext{
* Enfermeira.Prof. Assist. Dr. do Departamento de Enfermagem da Faculdade de Ciências Médicas da UNICAMP.
} 
Organizações internacionais e grupos de pesquisa destacam o pessoal de enfermagem como um grupo de risco em relação ao desenvolvimento de problemas dorsais e sugerem a utilização da ergonomia como estratégia fundamental de prevenção ${ }^{59,64,55}$.

A ergonomia é o estudo científico da relação entre o homem e seu ambiente de trabalho. Neste sentido, o termo ambiente abrange não apenas o meio propriamente dito em que o homem trabalha, mas também os instrumentos, os métodos e a organização deste trabalho. Em relação a tudo isto está ainda a natureza do próprio homem, o que inclui suas habilidades e capacidades psicofisiologicas, antropométricas e biomecânicas ${ }^{45}$.

O objetivo fundamental da ergonomia é contribuir para a satisfação das necessidades humanas no ambiente de trabalho, incluindo a promoção de saúde e de bem-estar ${ }^{27}$. Então, um dos pontos básicos para atingir este objetivo é a realização de uma análise cuidadosa do trabalho, voltada para a identificação dos fatores de incompatibilidade no contexto de trabalho e suas conseqüências para o indivíduo. Ao analisar de forma crítica e metodologica as situações de trabalho, a ergonomia visa a reorganizá-las de modo que se possa eliminar fontes de prejuízo, ou seja, eliminar aqueles elementos agressores que podem levar à perda parcial ou total de qualquer função vital, em curto, médio ou longo prazo ${ }^{24,48,63}$.

Nessa linha de pensamento, encontram-se hoje publicações voltadas para os problemas ergonômicos específicos de trabalhadores da enfermagem ${ }^{43,44}$. Assim, chegou-se à conclusão de que é fundamental o conhecimento, pelo pessoal de enfermagem, deste risco ocupacional e de alguns fatores relacionados com o aparecimento destas lesões, no contexto de trabalho da realidade brasileira. Acredita-se também que é urgente o estabelecimento de estratégias sistematizadas de prevenção, dentro de uma estrutura ergonômica.

Esse trabalho procura, então, discutir determinadas situações ergonômicas do trabalho que podem lesar a coluna vertebral, relacionando-as com as atividades de trabalho da equipe de enfermagem.

\section{2 - CONDIÇÕES QUE CAUSAM PROBLEMAS NO SISTEMA MÚSCULO-ESQUELÉTICO DAS COSTAS $E$ AS ATIVIDADES OCUPACIONAIS DA EQUIPE DE ENFERMAGEM}

Para muitos autores, os trabalhadores de enfermagem são especialmente suscetíveis a lesões nas costas pelo fato de terem que movimentar e transportar pacientes regularmente ${ }^{20,37,53,56,61}$. CATO et al. ${ }^{12}$ afirmaram que maioria dos episódios de lombalgia entre a equipe de enfermagem ocorre durante a movimentação de pacientes.

Contudo, HARBER et al..$^{28}$ ao investigarem fatores pessoais e de trabalho associados com a ocorrência de dores nas costas entre enfermeiras de hospitais, verificaram que as atividades que não envolvem o contato direto com pacientes podem ser as principais colaboradoras no desenvolvimento deste problema. Os autores também citaram que estas outras atividades ocupacionais são frequentemente ignoradas em pesquisas e em programas de treinamento.

Dessa forma, os questionamentos voltam-se para aspectos mais amplos, relacionados com o paciente, ambiente e organização do trabalho.

Para uma equipe de pesquisadores da Inglaterra, os problemas na coluna vertebral entre profissionais de enfermagem são causados por eventos cumulativos, incluindo o manuseio de pacientes ${ }^{59}$.

É importante salientar que estudos epidemiológicos gerais e estudiosos da coluna, têm relacionados os problemas da coluna vertebral com uma variedade de fatores ocupacionais, sendo que os mais citados são: trabalho físico pesado; levantamento e transporte manual de cargas; trabalho estático; atividades que exigem movimentos de flexão e rotação da coluna vertebral, entre outros $^{8,9,10,23,34,52,62}$.

Algumas considerações sobre algumas dessas condições serão apresentadas a seguir, procurando relacioná-las com as atividades ocupacionais da equipe de enfermagem.

\section{- Levantamento de peso excessivo}

Autores e instituições internacionais têm estado preocupados em avaliar a capacidade humana máxima para levantar e transportar cargas ${ }^{27,35,42}$. Em cada país existem, inclusive, leis especificas sobre o assunto.

No Brasil, o dispositivo que rege essa matéria é a norma regulamentadora $n^{\circ} 17$, que trata da Ergonomia do Ministério do Trabalho (Portaria $\mathrm{n}^{\mathrm{O}} 3.751$, de $\left.23 / 11 / 91\right)^{11}$.

A problemática da capacidade das mulheres também tem sido objeto de estudos ${ }^{26,55}$. A ORGANIZACIÓN INTERNACIONAL DEL TRABAJO ${ }^{42}$ recomenda que se limite o emprego de mulheres no transporte de cargas.

A idade é outro fator que é levado em conta quando se discute o levantamento de cargas. FINOCCHIARO et al. ${ }^{23}$ relataram que os discos intervertebrais suportam pressões diferentes em 
função da faixa etária, acrescentando que há um decréscimo na resistência destes discos com o envelhecimento.

E importante lembrar que entre cada corpo vertebral encontra-se uma estrutura excepcionalmente importante da coluna denominada disco intervertebral.

Os discos intervertebrais são compostos de uma massa gelatinosa localizada centralmente, o núcleo pulposo, que é rodeado por uma camada densa e resistente de fibrocartilagem, o anel fibroso, cujas fibras orientadas obliquamente previnem um deslocamento excessivo em qualquer direção ${ }^{49}$.

Esses discos são encarregados de amortecer cargas e pressões ao longo da coluna vertebral, ao mesmo tempo que permitem o movimento entre as vértebras. Segundo FINOCCHIARO et al. ${ }^{23}$, o disco é um sistema hidráulico completo que absorve choques, permite uma compressão transitória e, devido ao deslocamento do líquido dentro do continente elástico, permite o movimento.

Para explicar porque o disco intervertebral é uma estrutura propensa a uma degeneração precoce, CAILLIET $10 \mathrm{fez}$ as seguintes ponderações: "a vascularização do disco desaparece após a segunda década. Na terceira década, o disco agora nãovascularizado, é nutrido pela difusão de linfa através das placas terminais das vértebras em virtude das características físico-químicas de absorção do gel coloidal do núcleo. No processo de envelhecimento, o núcleo perde sua capacidade de fixar água, com uma perda adicional das propriedades de osmose e absorção".

Além da idade e sexo, atualmente sabe-se da existência de muitos outros fatores que devem ser considerados ao se desenvolverem critérios para determinar quais pesos máximos podem ser manipulados. Os mais citados são: localização e altura da carga em relação ao corpo; dimensões e formato da carga; freqüência e duração da tarefa; biotipologia e capacidade das pessoas envolvidas, assim como a postura corporal adotada durante os movimentos.

Pesquisadores têm utilizado as seguintes abordagens, para avaliar os riscos de problemas nas costas, quando cargas são levantadas: medida da pressão no disco intervertebral; modelos biomecânicos que determinam as forças na coluna lombar e a medida da pressão intra-abdominal. Desta forma, existem inúmeros trabalhos ergonômicos que procuram isolar cada uma das condições consideradas relevantes, utilizando-se de uma destas abordagens ou de medidas fisiologicas $7,16,36,40$.

Todos esses estudos são voltados para trabalhadores de indústrias e existem poucas pesquisas que procuram utilizar destas novas abordagens ergonômicas na questão da movimentação e transporte de pacientes ${ }^{21,25}$.

Inúmeros pesquisadores já têm demonstrado uma associação entre os problemas na coluna vertebral com a movimentação de pacientes acamados e com o excesso de esforço físico ao transportá-los $12,20,47,60$. Já se sabe inclusive que durante a realização desses procedimentos, o peso levantado por funcionários de enfermagem igualamse ou mesmo excedem as recomendações. Além disso, são realizados sob condições desfavoráveis, com uma equipe insuficiente e com equipamentos inadequados e sem manutenção ${ }^{2,22,57}$.

Para avaliar a manipulação de um paciente, além de todas as variáveis já citadas, deve-se pensar em fatores como disponibilidade de auxílios mecânicos; tipos de transportes; equipamentos utilizados; espaço físico disponível; e, finalmente, não se pode esquecer da segurança e conforto do próprio paciente.

Para finalizar, é importante ressaltar que o pessoal de enfermagem provavelmente está levantando cargas excessivas tanto ao movimentar e transportar pacientes, como quando mobiliza determinados materiais e equipamentos. Entre estes, pode-se citar: monitores, respiradores, caixas com frascos de soro, galões, pesos para tração e sacos de roupas.

\section{- Levantamento e manuseio de cargas de modo incorreto}

Outro fator que auxilia no desenvolvimento de lesões é o levantamento, na posição incorreta, de pesos abaixo dos limites permissíveis. Isto ocorre quando uma pessoa levanta uma carga com os membros inferiores estendidos e o dorso encurvado.

Alguns autores explicaram que esta posição provoca uma distensão musculoligamentar, argumentando que o modo mais errado de se levantar um peso é a utilização dos músculos do dorso para esta tarefa, pois estes devem ser considerados apenas como músculos posturais ${ }^{19,23,32}$.

Para GRANDJEAN ${ }^{27}$, as costas retas asseguram que as pressões nos discos intervertebrais sejam uniformemente distribuídas. Este autor afirmou também que manter as costas curvas e os joelhos retos provoca uma maior pressão nos discos da região lombar do que quando as costas estão retificadas e os joelhos flexionados. Este fato foi comprovado pelos estudos de NACHEMSON ${ }^{41}$, que demonstrou os efeitos da postura corporal e manuseio de cargas sobre a pressão no interior dos discos intervertebrais.

Atualmente, sabe-se que a carga sobre a coluna vertebral varia não só em função do peso 
suportado como também da posição do corpo e da distância do peso em relação à coluna.

Dentro desse contexto, CHAFFIN ${ }^{14,15}$ tem realizado pesquisas para avaliar os riscos de lesões na coluna utilizando modelos biomecânicos de trabalhadores efetuando atividades que envolvem $o$ manuseio de materiais. Como resultado destes estudos, o mesmo autor ${ }^{13}$ recomendou que as cargas devem ser levantadas o mais próximo possível do corpo, para reduzir a curvatura da coluna e a pressão nos músculos e discos intervertebrais.

Existem também outras orientações básicas que devem ser ministradas às pessoas que levantam e transportam cargas manualmente. Entre estas, pode-se citar: obter condições seguras do solo; posicionar os pés corretamente; evitar movimentos de torção do eixo vertical do corpo e segurar a carga usando totalmente as mãos ${ }^{3}$.

Seria importante observar se o trabalhador de enfermagem está manuseando cargas e pacientes de um modo correto e, também, obter informações se este pessoal recebeu treinamento quanto ảos métodos de trabalho que deverá utilizar para salvaguardar sua saúde e prevenir acidentes.

\section{- Levantamentos repetitivos}

Pressões não muito intensas sobre o disco intervertebral, porém repetitivas e freqüentes, podem ocasionar a aceleração da degeneração do mesmo, com perda das propriedades de amortecimento ${ }^{34}$.

COUTO $^{19}$ reforçou que o efeito do esforço físico crônico sobre a coluna vertebral é semelhante ao processo de envelhecimento: "há degeneração do disco, com diminuição do conteúdo de água do mesmo. Em conseqüência, as vértebras tornam-se aproximadas, e a coluna vertebral fica diminuída de tamanho".

Ao discutir aspectos fisiológicos e mecânicos do disco intervertebral, KAPANDJI ${ }^{31}$ fez as seguintes observações: "Se a aplicação de cargas e descargas sobre o disco são repetidas de um modo demasiado próximo no tempo, o disco não tem tempo para recuperar a sua espessura inicial. Também se cargas e descargas repetem-se de maneira demasiado prolongada, mesmo com a espera do tempo de recuperação necessário, o disco não recupera sua espessura inicial. Observa-se aqui um fenômeno de envelhecimento"

Dentro desse contexto, ressalta-se aqui que os trabalhadores de enfermagem além de manipular pacientes, também transportam e movimentam equipamentos e materiais durante suas atividades de trabalho.

\section{- Solicitações extraordinárias}

Ocorrem nas tarefas laborativas que determinam flexões, extensões, inclinações laterais e rotação da coluna vertebral, geralmente associadas com levantamento de pesos ${ }^{5,23}$.

Segundo CHAFFIN ${ }^{13}$, pesquisas recentes que se utilizam de modelos biomecânicos já conseguem revelar os riscos das forças compressoras e altas tensões musculares causados por inclinações laterais e movimentos de torção.

Para exemplificar essa condição na enfermagem, pode-se citar que quando funcionários preparam e movimentam bolsas para diálise peritoneal, assumem inúmeras posturas inadequadas que são agravadas pelo peso das bolsas.

\section{- Manutenção de uma postura por tempo prolongado}

Existem duas formas de trabalho, o estático e o dinâmico. $\mathrm{O}$ trabalho estático é altamente fatigante e, quando realizado freqüentemente, pode lesar articulações, tendões e ligamentos ${ }^{27,29}$. GRANDJEAN ${ }^{27}$ também enfatizou que a posição em pé em um mesmo local por longo tempo não só causa fadiga muscular como também um desconforto que é produzido por um retorno de sangue venoso insuficiente.

Dentro desse contexto, KNOPLICH ${ }^{34}$ fez a seguinte explanação: "A musculatura paravertebral é do tipo postural, preparada portanto para permanecer contraída como um todo durante grande período de tempo, com rodízio interno de suas fibras entre contratura e relaxamento, e desenvolvida o suficiente para manter a posição de equilíbrio na bipedestação do homem, função esta facilitada pelas curvaturas da coluna. Quando esta musculatura tem que desenvolver um esforço extra, no sentido de tornar o indivíduo mais ereto, ou quando tem que sustentar o tronco contra a ação da gravidade por mais tempo, manifesta-se a fadiga desta musculatura".

Ocasionais mudanças de postura durante a jornada de trabalho são recomendadas, tendo também como justificativa manter os discos em boas condições, pois, para serem nutridos, eles precisam estar submetidos a freqüentes mudanças de pressão $0^{27}$.

Uma das atividades que obriga o pessoal de enfermagem a permanecer na posição estática por tempo prolongado é a lavagem de materiais ${ }^{3}$.

\section{- Manutenção de posturas inadequadas}

Em relação aos efeitos de posturas defeituosas, já se sabe que elas produzem fadiga dos músculos 
dorsais e aumentam a carga sobre os discos intervertebrais ${ }^{13,17}$

Como no decorrer da jornada de trabalho um indivíduo pode assumir inúmeras posições diferentes, a análise e o registro da postura têm despertado a atenção de muitos pesquisadores. Por esta razão, desenvolveram-se vários métodos para quantificar a carga postural ${ }^{1,17,18,30,58}$.

Os poucos estudos realizados entre o grupo de enfermagem indicam que a postura mais adotada por este pessoal durante o trabalho é a estática em pé, o que representa um alto fator de risco $o^{6,38}$.

Os procedimentos que obrigam esse pessoal a permanecer na posição curvada ou em pé por tempo prolongado são: puncionar veia; ministrar banho no leito; realizar sondagem vesical, entre outros. Uma outra atividade que exige uma postura inadequada associada a um grande esforço físico, é o manuseio de autoclave.

\section{- Outras condições}

Certos estudiosos destacaram a importância dos microtraumas crônicos que a coluna vertebral sofre durante toda a vida. Além disso, são também citados o trauma direto e a tensão emocional ${ }^{23,34}$.

CAILLIET ${ }^{10}$ afirmou que uma pressão constante sobre o disco tem um papel preponderante na degeneração, justificando que a tensão física, na qual os músculos do corpo são mantidos em contração isométrica, exerce uma pressào sobre o disco, que acaba por diminuir sua capacidade embebitória. Acrescentou ainda que a contração dos músculos não permite que eles desempenhem a função de massagear o que prejudica a irrigação sangüínea local, e que a tensão emocional, por meio de seu componente muscular somático, provoca uma tensão muscular igual àquela provocada pelo trabalho físico.

É importante ressaltar que a equipe de enfermagem além de executar atividades que produzem tensão emocional, também está sujeita a causas não-ocupacionais.

\section{3 - CONSIDERAÇÕES FINAIS}

Ao concluir esse trabalho, não se pode deixar de frisar que todas as atividades ocupacionais mencionadas podem realmente produzir lesões dorsais, como comprovam as explanações ergonômicas citadas no decorrer do texto.

Acredita-se que é urgente a necessidade de estudos ergonômicos envolvendo as tarefas e as posturas adotadas pelo pessoal de enfermagem durante o trabalho. Através de técnicas ergonômicas observacionais, como o registro por meio de filmagem ou observação direta, deve-se analisar o tipo, freqüência e duração das posturas e atividades.

Dessa forma, espera-se que esse trabalho forneça subsidios que venham a contribuir no desenvolvimento de pesquisas que se utilizem de uma abordagem ergonômica, envolvendo equipamentos, mobiliários, pessoas e tarefas do ambiente hospitalar.

\section{REFERÊNCIAS BIBLIOGRÁFICAS}

1.AARAS, A.; WESTGAARD, R.H.; STRANDEN, E. Postural angles as an indicador of postural load and muscular injury in occupational work situations. Ergonomics, v. 31, n. 6, p. 915-33, 1988.

2.ALEXANDRE, N.M.C. Avaliação de determinados aspectos ergonômicos no transporte de pacientes. Ribeirào Preto, 1987. 114p. Dissertaçao(Mestrado) - Escola de Enfermagem de Ribeirão Preto, Universidade de São Paulo.

3.ALEXANDRE, N.M.C. et al. Aspectos ergonomicos e posturais em centro de material. Rev. Esc. Enf. USP, v. 26, n. 1, p 87-94, 1992.

4.BAKER, D.B.; LANDRIGAN, P.J. Occupationally related disorders. Med. Clin. North Am., v.74, n. 2, p. 441-60, 1990

5.BARREIRA, T.H.C. Um enfoque ergonômico para as posturas de trabalho. Rev. Bras. Saúde Ocup., v. 17, n.67, p. 61-71, 1989.

6.BATY, D.; STUBBS, D.A. Postural stress in geriatric nursing. Int. J. Nurs. Stud., v. 24, n. 4, p. 339-44, 1987.

7.BENDIX, T.; EID, S.E. The distance between the load and the body with three bi-manual lifting techniques. Applied Ergonomics, v. 14, n. 3, p. 185-92,1983.

8.BIERING-SORENSEN, F. A prospective study of low back pain in a general population. Scand. J. Rehab. Med., v. 15, n. 2 , p. $71-9,1983$.

9.BIGOS, S.J. et al. Back injuries in industry: a retrospective study. Spine, v. 11, n.3, p. 246-51, 1986.

10.CAILLIET, R. Sindromes dolorosas. Lombalgias. São Paulo, Ed. Manole, 1979.

11.CAMPANHOLE, A.; CAMPANHOLE, H.L. Consolidação das leis de trabalho e legislą̧̃o complementar. 83. ed. Sao Paulo, Atlas, 1991.

12.CATO, C.; OLSON, D.K.; STUDER, M. Incidence, prevalence, and variables associated with low back pain in staff nurses. AAOHN J., v. 37, n. 8, p. 321-7,1989.

13. CHAFFIN, D.B. Manual materials handling and the biomechanical basis for prevention of low back pain in industry - an overview. Am. Ind. Hyg. Assoc. J., v. 48, n. 12 , p. 989-96, 1987. 
14.CHAFFIN, D.B. Occupational biomechanics - a basis for workplace design to prevent musculoskeletal injuries. Ergonomics, v. 30, n. 2, p. 321-9, 1987.

15.CHAFFIN, D.B. Biomechanichal modelling of the low back during load lifting. Ergonomics, v. 31, n. 5, p. 685-97, 1988.

16.CIRIELLO, V.M. et al. The effects of task duration on psychophsically - determined maximum accetable weights ans forces. Ergonomics, v. 33, n. 2, p. 187.200, 1990.

17.CORLETT, E.N.; MANENICA, I. The effects and measurement of working postures. Applied Ergonomics, v. 11, n. 1, p. $7 \cdot 16,1980$.

18.CORLETT, E. N. ; MADELEY, S.J.; MANENICA, I. Posture targetting: a technique for recording working posture. Ergonomics, v. 22, n. 3, p.357-66,1979.

19. COUTO, H.A. Fisiologia do trabalho aplicada. Belo Horizonte, Ibérica, 1978.

20.CUST, G.; PEARSON, J.C.G. ; MAIR, A. The prevalence of low back pain in nurses. Int. Nurs. Rev., v. 19, n. 2, p. $169 \cdot 79,1972$.

21.DEHLIN, O.; JADERBERG, E. Perceived exertion during patient lifts. Scand. J. Rehab. Med., v. 14, p. 11-20, 1982.

22.DEHLIN, O.; LINDBERG, B. Lifting burden for a nursing aide during patient care in a geriatric ward. Scand. J. Rehab. Med., v. 7, p. 65-72, 1975.

23.FINOCCHIARO, J. et al. Manual de prevenção das lombalgias. Săo Paulo, Lex Ed., 1978.

24.FUNDAÇAO GETÚLIO VARGAS. Instituto Superior de Estudos e Pesquisas Psicossociais. Centro Brasileiro de Ergonomia e Cibernética. Análise Sociotecnológica. Rio de Janeiro, 1982.

25.GAGNON, M.; SICARD, C.; SIROIS, J.P. Evaluation of forces on the lumbo-sacral joint and assessment of work and energy transfer in nursing aides lifting patients. Ergonomics, v. 29, n. 3, p. 407.21, 1986.

26.GLOSS, D. Ergonomics and working woman. Occup. Health Saf., v. 48, n. 4 , p. 20-4, 1979.

27.GRANDJEAN, E. Lifting the task to the man. 4. ed. London, Taylor \& Francis, 1988.

28.HARBER. P. et al. Importance of non-patient transfer activities in nursing - related back pain: I. Questionnaire survey. J. Occup. Med., v. 29, n. 12, p. 967-70, 1987.

29.IIDA, I. Ergonomia : projeto e produção. São Paulo, Edgard Blucher, 1990.

30.KANT, I.; NOTREMANS, J.H.V.; BORM, P.J.A. Observations of working postures in garages using OWAS and consequent workload reduction recommendations. Ergonomies, v. 33, n. 2, p. 209-20, 1990.

31.KAPANDJI, I. A. Fisiologia articular. Esquemas comentados de mecanica humana. São Paulo, Ed. Manole, 1980. v.3.
32.KNOPLICH, J. Viva bem com a coluna que você tem. 12. ed. São Paulo, Ibrasa, 1987.

33.KNOPLICH, J. Agressðes posturais da profissão de desenhista Rev. Bras. Saúde Ocup., v. 15, n. 57, p. 55-8, 1987.

34.KNOPLICH, J. Ergonomia e coluna vertebral. Ars. Curandi v. 14, n. 6, p. $67-84,1981$.

35.KONZ, S. NIOSH lifting guidelines. Am. Ind. Hyg. Assoc. J. v. 43, n. 12 , p. $931-3,1982$.

36.LESKINEN, T.P.J. et al. A dynamic analysis of spinal compression with different lifting techiques. Ergonomics v. 26, n. 6, p. 595-604, 1983.

37.MARCHETTE, L. et al. Back injury: a preventable occupational hazard. Orthop. Nurs., v. 4, n. 6, p. 25-9, 1985.

38.MARZIALE, M.H.; MELO, M.R.C.; SILVA, E.M. A postura corporal adotada pela enfermeira durante a execução de seu trabalho. Rev. Bras. Saúde Ocup., v. 19, n. 73, p. 19 . $24,1991$.

39.MENDES, R. O impacto dos efeitos da ocupação sobre a saúde de trabalhadores. Rev. Saúde públ ., v. 22, n. 4, p. 311-26, 1988 .

40.MORRISSEY, S.J.; LIOU, Y.H. Maximum acceptable weights in load carriage. Ergonomics, v. 31, n. 2, p. 217-26, 1988.

41.NACHEMSON, A. The load on lumbar disks in different positions of the body. Clin.Orthop., n. 45, p. 107-22, 1966.

42.ORGANIZACION INTERNACIONAL DEL TRABAJO. Peso máximo de las cargas que puedem ser transportadas por un trabajador. Informe, v. 6, n. 2, p. 56-63, 1967.

43.OWEN, B.; GARG, A. Reducing back stress to nursing personnel: an ergonomic intervention in a nursing home. Ergonomics, v. 35, n. 11, p. 1353-75, 1992.

44. OWEN, B.; GARG, A. Back stress isn't part of the job. Amer. J. Nurs., v. , n. , p. 48-51, 1993.

45.PALMER, C. Ergonomia. Rio de janeiro, Ed. Fundação Getúlio Vargas, 1976.

46.PARNIAPOUR, $M$ et al. Environmentally induced disorders of the musculoskeletal system. Med. Clin. North Am. v. 74, n. 2 , p. $347-59,1990$.

47.RAISTRICK, A. Nurses with back pain - can the problem be prevented? Nurs. Times, v. 77, n. 14, p. 853-6, 1981.

48.RANIERE, T.M. Prevention of cumulative trauma injuries AAOHN J., v. 37, n. 6, p. 221.4, 1989.

49.RASCH, P.J.; BURKE, R.K. Cinesiologia e anatomia aplicada. 5. ed. Rio de Janeiro, Guanabara Koogan, 1977.

50.REBOUÇAS, A.J.A. et al. Insalubridade: morte lenta no trabalho. São Paulo, Oboré, 1989.

51.RIBEIRO, H.P.; LACAZ, F.A.C. (org.) De que adoecem e morrem os trabalhadores. São Paulo, DIESAT, 1985. 
52.RIIHIMAKI, H. Low back pain, its origin and risk indicators. Scand. J. Work Environ. Health, v. 17, n. 2, p. 81-90, 1991.

53.RODGERS, S. Back pain. Nurs. Times, v. 81, n. 3, p.24-6, 1985.

54.SKILLEN, L. Assessing occupational hazards. Can. Nurse, v. 84, n. 6, p. $25-8,1989$.

55.SNOOK, S.H.; CIRIELLO, V.M. Maximum weights and work loads acceptable to female workers. Occup. Health Nurs. v. 22, p. $11-20,1974$.

56.STUBBS, D.A. et al. Back pain research. Nurs. Times, v. 77, n. 20 , p. $857-8,1981$.

57.TUFFNELL, C. Taking the strain. NZ Nurs. J., v. 80, n. 4, p. 10.2, 1987.

58.TUNES, E.; GIL, H.J.C. Modelos de registro para a postura corporal em situaçoes funcionais. Rev. Bras. Saúde Ocup., v. 18, n. 69, p. 45-9, 1990.

59.UNIVERSITY OF SURREY. Ergonomics Research Unit. Back pain in nurses: summary and recommendations. Guildford, 1986.
60.VENNING, P.J.; WALTER, S.D.; STITT,L.W. Personal and jobrelated factors as determinants of incidence of back injuries among nursing personal. J. Occup. Med., v. 29, n. 10, p. $820-5,1987$.

61.VIDEMAN, T. et al. Low back pain in nurses and some loading factors of work. Spine, v. 9; n. 4. p. 400-4, 1984.

62.WALSH, K. et al. Occupational causes of low back pain. Scand. J. Work Environ. Health, v, 15, n. 1, p. 54-9, 1989.

63.WICK, J.L. The role of ergonomics in the elimination and prevention of work-related musculoskeletal problems. Orthop. Nurs., v. 8, n. 1, p. 41-2, 1989.

64.WORLD HEALTH ORGANIZATION. Identification and control of work-related diseases . Geneva, 1985. (Techn. Rep. Ser. 714)

65.WORLD HEALTH ORGANIZATION. Occupational hazards in hospitals. Copenhagem, 1981. (EURO Reports and Studies 80). 